Research Article

\title{
Treating Clinically Node-Negative Insular Thyroid Carcinoma without Prophylactic Central Compartment Neck Dissection Is Associated with Decreased Survival Regardless of $T$ Staging and Administration of Radioactive Iodine Therapy: The First Evidence
}

\author{
Peng-Cheng Yu, ${ }^{1,2}$ Xiao Shi, ${ }^{1,2}$ Ben Ma, ${ }^{1,2}$ Cui-Wei Li, ${ }^{1,2}$ Li-Cheng Tan, ${ }^{1,2}$ Wei-Ping Hu, ${ }^{3}$ \\ Yu Wang, ${ }^{1,2}$ Wen-Jun Wei $\mathbb{D}^{1,2}$ Yu-Long Wang $\mathbb{D}^{1,2}$ and Qing-Hai Ji $\mathbb{D}^{1,2}$ \\ ${ }^{1}$ Department of Head and Neck Surgery, Fudan University Shanghai Cancer Center, Shanghai, 200032, China \\ ${ }^{2}$ Department of Oncology, Shanghai Medical College, Fudan University, Shanghai, 200032, China \\ ${ }^{3}$ Department of Internal Medicine, Shanghai Medical College, Fudan University, Shanghai, 200032, China
}

Correspondence should be addressed to Wen-Jun Wei; kakarwen@163.com, Yu-Long Wang; wylheadneck@sina.com, and Qing-Hai Ji; jq_hai@126.com

Received 2 January 2019; Revised 7 April 2019; Accepted 30 August 2019; Published 16 October 2019

Academic Editor: Lodovico Rosato

Copyright (c) 2019 Peng-Cheng Yu et al. This is an open access article distributed under the Creative Commons Attribution License, which permits unrestricted use, distribution, and reproduction in any medium, provided the original work is properly cited.

\begin{abstract}
For the rare but aggressive insular thyroid carcinoma (ITC), there's no clear evidence to determine whether prophylactic central compartment neck dissection (CCND) is necessary for $\mathrm{cN} 0$ disease. This study provides the first evidence that treating $\mathrm{cN} 0$ ITC without prophylactic CCND is associated with decreased survival regardless of $T$ staging and administration of RAI therapy. Background. Regarding the rare but aggressive insular thyroid carcinoma (ITC), the value of prophylactic central compartment neck dissection (CCND) for clinically node-negative (cN0) disease is unclear. We aimed to provide the first evidence. Methods. N0 and pN1a ITC patients were identified from the Surveillance, Epidemiology, and End Results database. These patients were divided into thyroid-surgery + CCND group ( $\mathrm{pN} 0 / \mathrm{pN} 1$ a patients confirmed by CCND) and thyroid-surgery group (cN0 patients without CCND). Differences in overall survival (OS) and disease-specific survival (DSS) between the two groups were evaluated. Subgroup analyses were also conducted. Results. Of the overall 112 patients, 44 (39.3\%) received CCND. On multivariate analyses, the lobectomy \pm isthmusectomy/total-thyroidectomy (Lob/TT) group demonstrated poorer OS and DSS than the Lob/TT + CCND group $(P<0.05)$. When we separately analyzed patients treated by TT, multivariate analyses showed the TT group still revealed compromised OS and DSS than the TT + CCND group $(P<0.05)$. Furthermore, absence of CCND independently predicted decreased OS no matter whether radioactive iodine (RAI) was administered. Similar results were obtained for T3/T4 patients. Moreover, for T1/T2 patients receiving CCND, 0/12 died during the study period, while for T1/T2 patients without CCND, 8/23 (34.8\%) died, 5/23 (21.7\%) due to ITC. Conclusion. Regardless of T staging and RAI treatment, cN0-ITC patients without CCND had decreased survival compared with pN0/pN1a patients receiving CCND. Therefore, if a cN0 patient is diagnosed with ITC, prophylactic CCND may be considered as a secondary procedure (postoperatively diagnosed) or a primary procedure (preoperatively/intraoperatively diagnosed). Prospective studies are expected to validate the conclusion.
\end{abstract}

\section{Introduction}

First described by Carcangiu et al. in 1984 [1], insular thyroid carcinoma (ITC) is a rare but aggressive thyroid malignancy categorized as the most common subtype of poorly differentiated thyroid carcinoma (PDTC) [2-4]. Data from both small series and population-level databases show that ITC is associated with larger tumor size, higher rate of 
extrathyroidal extension (ETE), nodal involvement, and distant metastasis [2, 5-8].

The value of prophylactic central compartment neck dissection (CCND) in clinically node-negative (cN0) differentiated thyroid cancer (DTC) has been a hot topic for decades but is still a matter of debate. With regard to PDTC found postoperatively, the previous National Comprehensive Cancer Network (NCCN) guideline (2017 version 2) lists CCND as a consideration for $\mathrm{cN} 0$ patients [9]. However, this statement is deleted in the latest version (2018 version 1) [10]. On the other hand, in the 2015 American Thyroid Association (ATA) guideline, indications of prophylactic CCND for ITC or PDTC are not separately distinguished from classic DTCs [3].

Although the 2015 ATA guideline recommends prophylactic CCND for T3/T4 cN0 patients, lobectomy without CCND is regarded appropriate for $\mathrm{cN} 0$ patients with $\mathrm{T} 1 / \mathrm{T} 2$ tumors [3]. Besides, several scholars also reported that with the use of radioactive iodine (RAI) ablation, total thyroidectomy without CCND could also achieve a low locoregional recurrence rate or low postoperative thyroglobulin levels in $\mathrm{cN} 0$ patients $[11,12]$.

Due to the aggressive behavior of the insular subtype, whether cN0 ITC necessitates CCND, especially for T1/T2 patients or those undergoing RAI therapy, deserves further attention in clinical practice. However, given the paucity of data, this problem has never been investigated. In this study, we sought to explore this issue using data from the Surveillance, Epidemiology, and End Results (SEER) database.

\section{Materials and Methods}

2.1. Data Source and Study Population. Using the SEER database, the eligible patients were identified according to the following selection criteria: (1) diagnosed with ITC as the first malignancy using the International Classification of Diseases for Oncology, 3rd edition (ICD-O-3) code 8337; (2) diagnosed between 1999 and 2014, as the first ITC patient recorded in the SEER program was diagnosed in 1999; (3) received thyroid surgery; (4) without distant metastasis at diagnosis; (5) with N0 or pN1a record of diagnosis; (6) with definite record of $T$ staging and known tumor size. We excluded patients without information on whether CCND was performed and those less than 18 years. The selection process is presented as a flow diagram (Supplementary Figure S1). In total, 112 patients were identified and constituted the study cohort.

Institutional Review Board (IRB) approval is not necessary for this study, because the SEER program is a publicly available cancer database with deidentified data.

2.2. Variables and Outcomes. Data extracted for each case included age at diagnosis, sex, race, $T$ and $N$ stages, multifocality, extrathyroidal extension (ETE), tumor size, thyroid surgery, radiation, and presence/absence of CCND. As small ITCs were sometimes found after lobectomy, in the present study, thyroid surgery included both lobectomy \pm isthmusectomy (Lob) and total thyroidectomy (TT) to better reflect the real clinical situation. Radiation was divided into three categories: "no evidence," "external beam radiotherapy (EBRT)," and "RAI," just like the previous studies $[2,5]$. As only four patients received chemotherapy, this parameter was not included in the analyses. The $T$ and $N$ staging were identified according to the American Joint Committee (AJCC) eighth edition.

The N0 patients included pNO patients confirmed by CCND and cN0 patients without prophylactic CCND. It is worth noting that, in the SEER database, if a patient has a pathologic stage, the record of clinical stage will be covered and not available to users. Consequently, the $\mathrm{cN}$ staging ( $\mathrm{cN0} / \mathrm{cN} 1)$ of $\mathrm{pN0}$ and pN1a patients was unknown, the CCND of whom might include both prophylactic CCND and therapeutic CCND. Subsequently, the overall cohort was divided into the thyroid-surgery + CCND group ( $\mathrm{pN} 0 / \mathrm{pN} 1 \mathrm{a}$ patients confirmed by CCND) and thyroid-surgery group (cN0 patients without CCND).

Overall survival (OS) and disease-specific survival (DSS), respectively, defined as the interval from initial pathologic diagnosis to the date of all-cause or ITC-specific death were compared between the two groups.

2.3. Statistical Analysis. The differences of baseline characteristics across the two groups were compared by chisquared test or Fisher's exact test, as appropriate. The survival curves were plotted by Kaplan--Meier estimates, and the differences were compared by log-rank tests. Multivariate Cox proportional hazards regression models were employed to identify the prognostic factors after adjusting for potential confounders, and the results were presented as hazard ratios (HRs) with 95\% confidence intervals (CI). To better evaluate the prognostic relevance of CCND, for all multivariate analyses (MVA), two types of Cox regression models were parallelly performed. The Type-A model was defined as the Cox regression model including comprehensive baseline variables (age at diagnosis, sex, race, multifocality, ETE, tumor size, thyroid surgery, radiation, and CCND), $T$ stage was not incorporated because the two factors, tumor size and ETE, which constituted the Tstaging were both included in the model. The Type- $\mathrm{B}$ model included the best subsets of covariates identified by the smallest Akaike Information Criterion (AIC) value, which reflected the minimal loss of information [13-15].

Log-rank tests and multivariate Cox analyses were conducted by SPSS Statistics v23.0 (SPSS Inc., Chicago, IL). Kaplan-Meier survival plots were generated by GraphPad Prism 7.0 (GraphPad Software, San Diego, CA). The AIC values were calculated by $\mathrm{R}$ version 3.4.3 (R-Foundation for Statistical Computing, Vienna, Austria). Statistical significance was defined as a two-tailed $P$ value $<0.05$.

\section{Results}

3.1. Patient Characteristics. Of the 112 patients, the median follow-up length was 67 months (range: $2-167$ months) and the median age at diagnosis was 58 years (range: 21-94). $64.3 \%$ of patients $(n=72)$ had tumors larger than $4 \mathrm{~cm}$, nearly three fourths of the tumors were unifocal $(n=83$, 
$74.1 \%)$, and approximately one third $(n=38,33.9 \%)$ had ETE. 98 (87.5\%) patients underwent TT, and $70(62.5 \%)$ received RAI therapy (Table 1 ).

102 patients were recorded as N0, 34 were $\mathrm{pN} 0$ receiving CCND, and 68 patients were cN0 not receiving CCND. 10 patients were diagnosed with $\mathrm{pN} 1 \mathrm{a}$ disease (possible reasons were discussed in Discussion), and none of these patients had level VII (upper mediastinal) metastases. The pN0 and pN1a patients undergoing CCND constituted the Lob/ TT + CCND group $(n=44)$. The $68 \mathrm{cN} 0$ patients without CCND constituted the Lob/TT group. Baseline characteristics across the two groups are also shown in Table 1. A significantly higher proportion of patients with ETE was observed in the Lob/TT + CCND group $(P=0.038)$.

3.2. CCND versus No-CCND in the Overall Cohort. The survival difference between the Lob/TT + CCND group and the Lob/TT group was investigated for the overall cohort. Among 44 patients in the Lob/TT + CCND group, 5 (11.4\%) died during the study period, 3 (6.8\%) of which were due to ITC. Among 68 patients in the Lob/TT group, we observed $25(35.8 \%)$ deaths and $16(23.5 \%)$ were due to ITC (Figure 1). The log-rank test showed that the Lob/TT + CCND group had a significantly improved OS compared with the Lob/TT group $(P=0.048)$. No significant difference in DSS was observed in the log-rank test $(P=0.078)$.

As we mentioned in Materials and Methods, two types of MVA were performed. (i) In the Type-A Cox models, the Lob/TT group was independently associated with worse OS $(\mathrm{HR}=4.640$ (95\% CI 1.576-13.659), $P=0.005)$ and DSS $(\mathrm{HR}=5.707$ (95\% CI 1.362-23.907), $P=0.017)$ compared with the Lob/TT + CCND group (Table 2). (ii) The smallest AIC values (214.9 for OS; 143.3 for DSS) were obtained when we incorporated age at diagnosis, sex, multifocality, ETE, and CCND into the Type-B Cox models for both endpoints; the Lob/TT group still had significantly poorer prognosis (OS : $\mathrm{HR}=4.408$ (95\% CI 1.510-12.865), $P=0.007$; DSS : $\mathrm{HR}=5.523(95 \%$ CI 1.342-22.730), $P=0.018)$ (Supplementary Table S1).

3.3. CCND versus No-CCND for Patients Treated with TT. Subsequently, we focused on patients treated with TT. (i) In the Type-A Cox models, the TT group demonstrated significantly inferior OS ( $\mathrm{HR}=4.175$ (95\% CI 1.325-13.158), $P=0.015)$ and DSS (HR $=5.087$ (95\% CI 1.089-23.751), $P=0.039$ ) compared with the TT + CCND group (Table 3 ). (ii) In the Type-B models, the significantly higher mortality risk of the TT group was also observed (OS : HR $=4.242(95 \%$ CI 1.376-13.082), $P=0.012 ; \quad \mathrm{DSS}: \mathrm{HR}=5.272 \quad(95 \%$ CI 1.170-23.767), $P=0.030$ ) (Supplementary Table S2).

3.4. CCND versus No-CCND according to Whether RAI Therapy Was Administered. For patients who underwent TT and RAI, absence of CCND still predicted compromised survival in both Type-A (OS : HR =7.137 (95\% CI 1.35437.636), $P=0.021$; DSS : $\mathrm{HR}=15.796$ (95\% CI 1.380-180.789), $P=0.026)$ and Type-B Cox models incorporating age at diagnosis, multifocality, ETE, and CCND determined by the smallest AIC (OS : HR $=7.350$ (95\% CI 1.440-37.520), $P=0.016 ; \quad$ DSS $: H R=14.933 \quad(95 \%$ CI $1.442-154.649)$, $P=0.023)$, as shown in Supplementary Tables S3 and S4.

For patients who did not receive RAI ablation, the prognostic difference failed to meet statistical significance in Type-A Cox regression models (OS: $\mathrm{HR}=3.951$ (95\% CI $0.803-19.945), P=0.082$; DSS $: \mathrm{HR}=2.977$ (95\% CI $0.786-$ 15.539), $P=0.095$ ) (Supplementary Table S5). However, absence of CCND still turned out to be an independent adverse prognostic factor for OS (OS : $\mathrm{HR}=3.972$ (95\% CI 1.103-14.155), $P=0.035)$ in the Type-B model controlling for variables identified by the smallest AIC, as shown in Supplementary Table S6.

3.5. CCND versus No-CCND according to T Stage. For patients with T3/T4 disease, similar results were obtained. As shown in Supplementary Tables S7 and S8, both the two types of MVA confirmed the increased risk of mortality for patients without CCND (Type-A : OS: HR=3.659 (95\% CI 1.196-11.196), $P=0.023$; DSS : HR $=4.414$ (95\% CI $1.084-$ 17.967), $\quad P=0.038 ;$ Type-B : OS: $\mathrm{HR}=3.318 \quad(95 \% \quad \mathrm{CI}$ 1.107-9.946), $P=0.032$; DSS $: \mathrm{HR}=4.178$ (95\% CI $1.040-$ 16.780), $P=0.044)$.

For patients with T1/T2 disease, there were no significant differences in age at diagnosis, sex, race, multifocality, thyroid surgery, and radiation (Supplementary Table S9). However, during the study period with a median follow-up time of 83 months (range: 3-167 months), none of the 12 patients died $(0 / 12,0.0 \%)$ in the Lob/TT + CCND group, while $8 / 23$ (34.8\%) died in the Lob/TT group and $5(21.7 \%)$ were due to ITC (Figures 2(a) and 2(b)). Multivariate analyses could not be performed because there was no death in the Lob/TT + CCND group.

Furthermore, we focused on $\mathrm{T} 1 / \mathrm{T} 2$ patients undergoing TT plus RAI and all-cause mortality in the TT + CCND group and TT group was $0 / 9(0.0 \%)$ and 6/17 (35.3\%), respectively, while disease-specific mortality in the two groups was $0 / 9(0.0 \%)$ and $4 / 17(23.5 \%)$, respectively (Figures 2(c) and $2(\mathrm{~d}))$. Similarly, MVA could not be conducted due to the lack of death $(n=0)$ in the TT + CCND group.

\section{Discussion}

Biologically and morphologically, ITC occupies an intermediate position between well-differentiated thyroid carcinoma (WDTC) and anaplastic (undifferentiated) thyroid carcinoma (ATC) [2]. Kazaure et al. firstly reported that the insular subtype was independently associated with a poor survival compared to WDTC using the SEER database [5]. Pezzi et al. reported the 5- and 10-year survival rates of ITC were, respectively, 57\% and 30\%, significantly worse than papillary thyroid cancer (PTC) with the 5- and 10-year survival rates of $94 \%$ and $88 \%$ using the National Cancer Database (NCDB) [2].

Nevertheless, neither the 2015 ATA nor the latest NCCN guideline pays special attention to the indication of prophylactic CCND for ITC patients $[3,10]$, possibly due to the 
TABLE 1: Baseline characteristics of the overall cohort $(N=112)$.

\begin{tabular}{|c|c|c|c|c|}
\hline Characteristics & Overall cohort $n=112$ & Lob/TT + CCND group $N=44$ & Lob/TT group $N=68$ & $P$ \\
\hline Age at diagnosis & & & & 0.360 \\
\hline Median (range) & $58(21-94)$ & $55(21-94)$ & $60(21-88)$ & \\
\hline$<55$ & $45(40.2 \%)$ & $20(45.5 \%)$ & $25(36.8 \%)$ & \\
\hline$\geq 55$ & $67(59.8 \%)$ & $24(54.5 \%)$ & $43(63.2 \%)$ & \\
\hline Sex & & & & 0.174 \\
\hline Female & $65(58.0 \%)$ & $29(65.9 \%)$ & $36(52.9 \%)$ & \\
\hline Male & $47(42.0 \%)$ & $15(34.1 \%)$ & $32(47.1 \%)$ & \\
\hline Race & & & & 0.703 \\
\hline White & $87(77.7 \%)$ & $34(77.3 \%)$ & $53(77.9 \%)$ & \\
\hline Black & $15(13.4 \%)$ & $5(11.4 \%)$ & $10(14.7 \%)$ & \\
\hline Others & $10(8.9 \%)$ & $5(11.4 \%)$ & $5(7.4 \%)$ & \\
\hline Tumor size & & & & 0.773 \\
\hline$\leq 4 \mathrm{~cm}$ & $40(35.7 \%)$ & $15(34.1 \%)$ & $25(36.8 \%)$ & \\
\hline$>4 \mathrm{~cm}$ & $72(64.3 \%)$ & $29(65.9 \%)$ & $43(63.2 \%)$ & \\
\hline Multifocal & & & & 0.423 \\
\hline No & $83(74.1 \%)$ & $34(77.3 \%)$ & $49(72.1 \%)$ & \\
\hline Yes & $19(17.0 \%)$ & $8(18.2 \%)$ & $11(16.2 \%)$ & \\
\hline Unknown & $10(8.9 \%)$ & $2(4.5 \%)$ & $8(11.8 \%)$ & \\
\hline Extrathyroidal extension & & & & 0.038 \\
\hline No & $74(66.1 \%)$ & $24(54.5 \%)$ & $50(73.5 \%)$ & \\
\hline Yes & $38(33.9 \%)$ & $20(45.5 \%)$ & $18(26.5 \%)$ & \\
\hline AJCC 8th $T$ staging & & & & 0.465 \\
\hline $\mathrm{T} 1 / \mathrm{T} 2$ & $35(31.3 \%)$ & $12(27.3 \%)$ & $23(33.8 \%)$ & \\
\hline $\mathrm{T} 3 / \mathrm{T} 4$ & $77(68.7 \%)$ & $32(72.7 \%)$ & $45(66.2 \%)$ & \\
\hline Surgery of thyroid gland & & & & 0.380 \\
\hline Lob & $14(12.5 \%)$ & $4(9.1 \%)$ & $10(14.7 \%)$ & \\
\hline $\mathrm{TT}$ & $98(87.5 \%)$ & $40(90.9 \%)$ & $58(85.3 \%)$ & \\
\hline Radiation & & & & 0.475 \\
\hline RAI & $70(62.5 \%)$ & $30(68.2 \%)$ & $40(58.8 \%)$ & \\
\hline EBRT & $12(10.7 \%)$ & $3(6.8 \%)$ & $9(13.2 \%)$ & \\
\hline No evidence & $30(26.8 \%)$ & $11(25.0 \%)$ & $19(27.9 \%)$ & \\
\hline
\end{tabular}

Lob refers to lobectomy \pm isthmusectomy; TT = total thyroidectomy; CCND = central compartment neck dissection; EBRT=external beam radiotherapy; $\mathrm{RAI}=$ radioactive iodine .

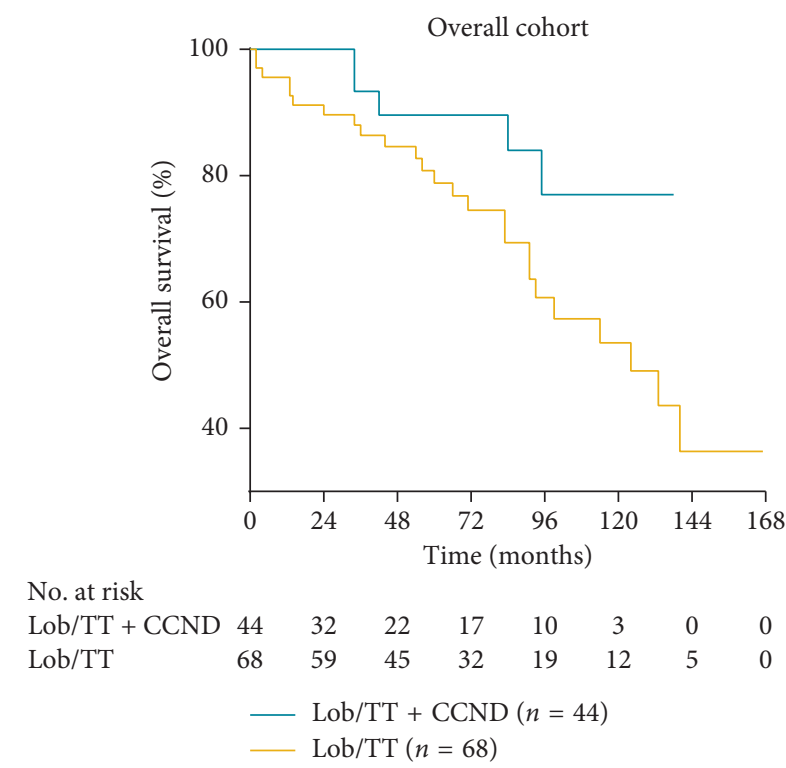

(a)

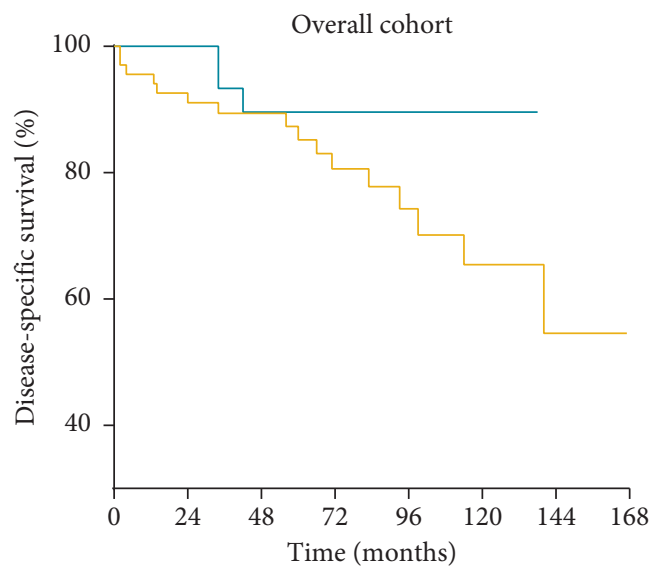

No. at risk

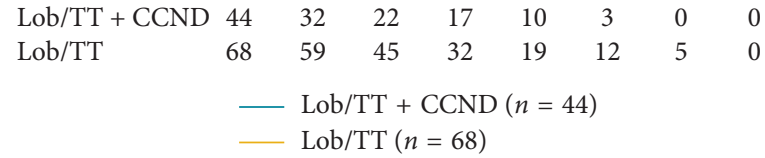

(b)

FIGURE 1: Kaplan-Meier survival plots presenting (a) OS and (b) DSS of the Lob/TT group and Lob/TT + CCND group for patients in the overall study cohort $(99 \times 43 \mathrm{~mm}(300 \times 300 \mathrm{DPI}))$. 
TABLE 2: Type-A multivariate Cox regression models investigating the factors associated with OS and DSS in the overall cohort ( $N=112)$.

\begin{tabular}{|c|c|c|c|c|}
\hline \multirow{2}{*}{ Variables } & \multicolumn{2}{|c|}{ OS } & \multicolumn{2}{|c|}{ DSS } \\
\hline & HR $(95 \% \mathrm{CI})$ & $P$ & $\mathrm{HR}(95 \% \mathrm{CI})$ & $P$ \\
\hline \multicolumn{5}{|l|}{ Age at diagnosis } \\
\hline$<55$ & Ref & & Ref & \\
\hline$\geq 55$ & $7.663(2.386-24.607)$ & 0.001 & $6.630(1.557-28.234)$ & 0.011 \\
\hline \multicolumn{5}{|c|}{ 年 } \\
\hline Female & Ref & & Ref & \\
\hline Male & $2.619(1.120-6.123)$ & 0.026 & $2.798(0.899-8.707)$ & 0.076 \\
\hline \multicolumn{5}{|l|}{ Race } \\
\hline White & Ref & & Ref & \\
\hline Black & $0.897(0.225-3.578)$ & 0.877 & $2.322(0.527-10.221)$ & 0.265 \\
\hline Others & $0.869(0.191-3.965)$ & 0.856 & $1.158(0.230-5.841)$ & 0.859 \\
\hline \multicolumn{5}{|l|}{ Tumor size } \\
\hline$\leq 4 \mathrm{~cm}$ & Ref & & Ref & \\
\hline$>4 \mathrm{~cm}$ & $1.348(0.553-3.279)$ & 0.512 & $1.799(0.564-5.747)$ & 0.321 \\
\hline \multicolumn{5}{|l|}{ Multifocal } \\
\hline No & Ref & & Ref & \\
\hline Yes & $3.783(1.214-11.791)$ & 0.022 & $6.795(1.565-29.496)$ & 0.011 \\
\hline Unknown & $2.579(0.717-9.274)$ & 0.147 & $1.535(0.314-7.516)$ & 0.597 \\
\hline \multicolumn{5}{|c|}{ Extrathyroidal extension } \\
\hline No & Ref & & Ref & \\
\hline Yes & $5.602(2.302-13.635)$ & $<0.001$ & $11.996(3.374-42.652)$ & $<0.001$ \\
\hline \multicolumn{5}{|c|}{ Surgery of thyroid gland } \\
\hline Lob & Ref & & Ref & \\
\hline $\mathrm{TT}$ & $0.567(0.160-2.013)$ & 0.380 & $0.412(0.081-2.100)$ & 0.286 \\
\hline \multicolumn{5}{|l|}{ Radiation } \\
\hline RAI & Ref & & Ref & \\
\hline EBRT & $0.503(0.111-2.291)$ & 0.375 & $0.955(0.185-4.935)$ & 0.956 \\
\hline No evidence & $1.825(0.692-4.812)$ & 0.224 & $3.542(0.959-13.079)$ & 0.058 \\
\hline \multicolumn{5}{|l|}{ CCND } \\
\hline Yes & Ref & & Ref & \\
\hline No & $4.640(1.576-13.659)$ & 0.005 & $5.707(1.362-23.907)$ & 0.017 \\
\hline
\end{tabular}

OS = overall survival; DSS = disease-specific survival; $\mathrm{HR}=$ hazard ratio; $\mathrm{CI}=$ confidence interval; $\mathrm{Ref}=$ reference.

TABLE 3: Type-A multivariate Cox regression models investigating the factors associated with OS and DSS for patients treated with TT $(N=98)$.

\begin{tabular}{|c|c|c|c|c|}
\hline \multirow{2}{*}{ Variables } & \multicolumn{2}{|c|}{ OS } & \multicolumn{2}{|c|}{ DSS } \\
\hline & HR $(95 \%$ CI) & $P$ & HR (95\% CI) & $P$ \\
\hline \multicolumn{5}{|c|}{ Age at diagnosis } \\
\hline$<55$ & Ref & & Ref & \\
\hline$\geq 55$ & $7.794(1.977-30.724)$ & 0.003 & $9.541(1.472-61.830)$ & 0.018 \\
\hline \multicolumn{5}{|c|}{ (1) } \\
\hline Female & Ref & & Ref & \\
\hline Male & $2.698(1.032-7.056)$ & 0.043 & $3.408(0.935-12.421)$ & 0.063 \\
\hline \multicolumn{5}{|l|}{ Race } \\
\hline White & Ref & & Ref & \\
\hline Black & $1.092(0.234-5.098)$ & 0.911 & $3.489(0.601-20.250)$ & 0.164 \\
\hline Others & $1.019(0.218-4.759)$ & 0.981 & $1.413(0.266-7.508)$ & 0.685 \\
\hline \multicolumn{5}{|l|}{ Tumor size } \\
\hline$\leq 4 \mathrm{~cm}$ & Ref & & Ref & \\
\hline$>4 \mathrm{~cm}$ & $1.129(0.411-3.096)$ & 0.815 & $1.938(0.508-7.407)$ & 0.333 \\
\hline \multicolumn{5}{|l|}{ Multifocal } \\
\hline No & Ref & & Ref & \\
\hline Yes & $3.984(1.226-12.947)$ & 0.022 & $8.837(1.820-42.913)$ & 0.007 \\
\hline Unknown & $3.716(0.817-16.911)$ & 0.089 & $3.052(0.376-24.808)$ & 0.297 \\
\hline Extrathyroida & & & & \\
\hline
\end{tabular}


TABLE 3: Continued.

\begin{tabular}{|c|c|c|c|c|}
\hline \multirow{2}{*}{ Variables } & \multicolumn{2}{|c|}{ OS } & \multicolumn{2}{|c|}{ DSS } \\
\hline & HR $(95 \% \mathrm{CI})$ & $P$ & $\operatorname{HR}(95 \% \mathrm{CI})$ & $P$ \\
\hline No & Ref & & Ref & \\
\hline Yes & $4.264(1.662-10.940)$ & 0.003 & $8.427(2.089-34.003)$ & 0.003 \\
\hline \multicolumn{5}{|l|}{ Radiation } \\
\hline RAI & Ref & & Ref & \\
\hline EBRT & $0.931(0.198-4.373)$ & 0.928 & $2.394(0.430-13.334)$ & 0.319 \\
\hline No evidence & $1.392(0.501-3.869)$ & 0.525 & $2.720(0.660-11.214)$ & 0.166 \\
\hline \multicolumn{5}{|l|}{ CCND } \\
\hline Yes & Ref & & Ref & \\
\hline No & $4.175(1.325-13.158)$ & 0.015 & $5.087(1.089-23.751)$ & 0.039 \\
\hline
\end{tabular}

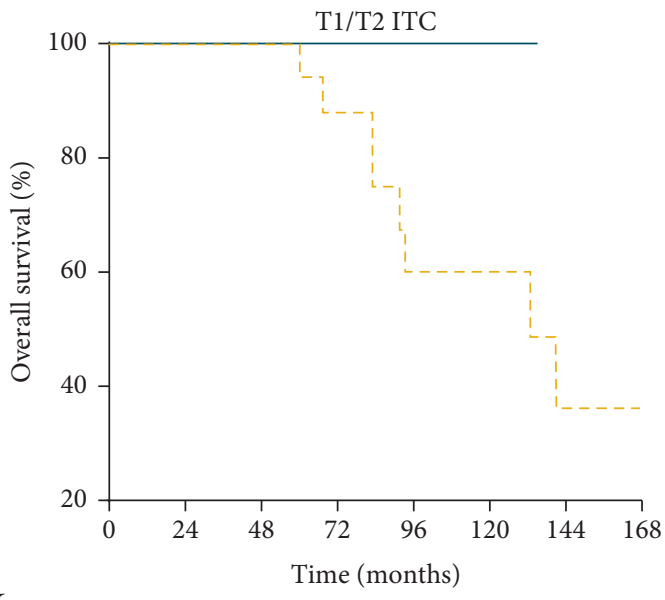

$\begin{array}{lccccc}\begin{array}{l}\text { No. at risk } \\ \text { Lob/TT + CCND } \\ \text { Lob/TT }\end{array} & 12 & 8 & 6 & 5 & 3 \\ & 23 & 22 & 17 & 15 & 8 \\ & & \text { Lob/TT + CCND }(n=12) \\ & & \text { Lob/TT }(n=23)\end{array}$

(a)

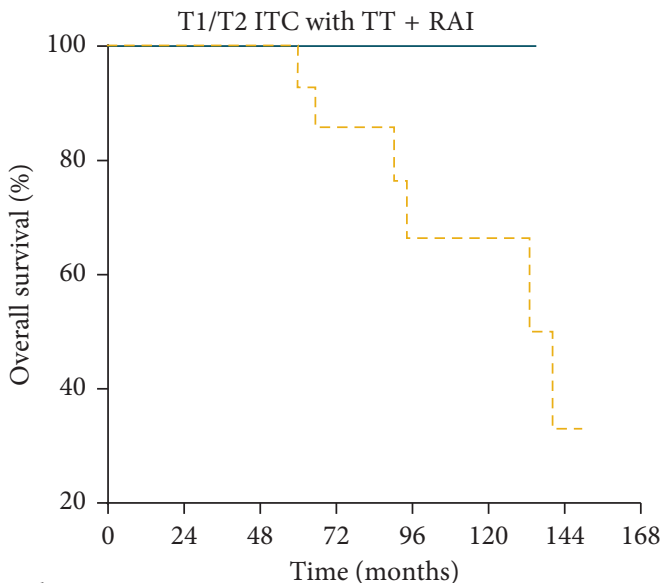

No. at risk

$\begin{array}{lllllllll}\mathrm{TT}+\mathrm{CCND} & 9 & 6 & 5 & 4 & 2 & 1 & 0 & 0\end{array}$

$\begin{array}{lllllllll}\text { TT } & 17 & 17 & 14 & 12 & 7 & 4 & 2 & 0\end{array}$

$-\mathrm{TT}+\operatorname{CCND}(n=9)$

--- TT $(n=17)$

(c)

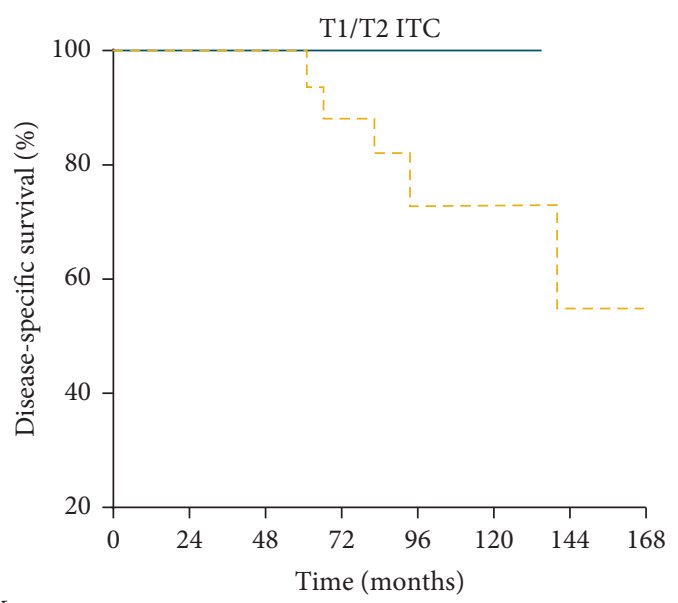

No. at risk

$\begin{array}{lcccccccc}\text { Lob/TT + CCND } & 12 & 8 & 6 & 5 & 3 & 1 & 0 & 0 \\ \text { Lob/TT } & 23 & 22 & 17 & 15 & 8 & 5 & 3 & 0\end{array}$

- Lob/TT + CCND $(n=12)$

-. $\operatorname{Lob} / \mathrm{TT}(n=23)$

(b)

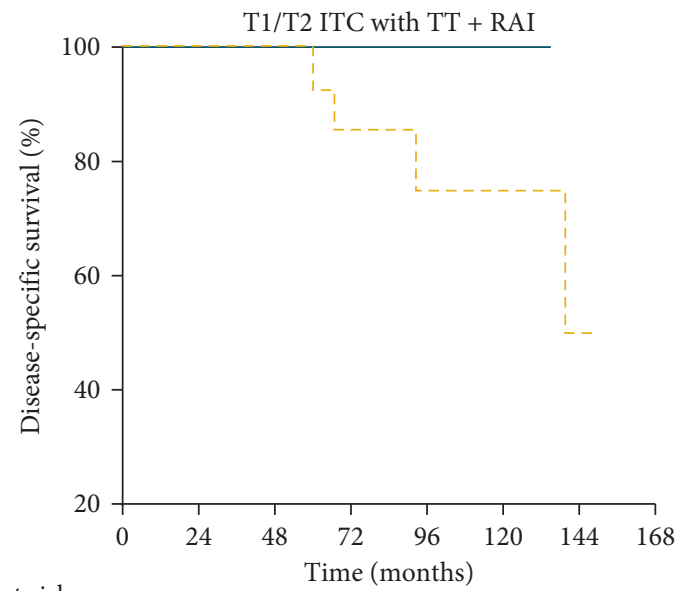

No. at risk

$\begin{array}{lllllllll}\mathrm{TT}+\mathrm{CCND} & 9 & 6 & 5 & 4 & 2 & 1 & 0 & 0\end{array}$

$\mathrm{TT}$

$\begin{array}{llllllll}17 & 17 & 14 & 12 & 7 & 4 & 2 & 0\end{array}$

$-\mathrm{TT}+\mathrm{CCND}(n=9)$

$--\operatorname{TT}(n=17)$

(d)

FIGURE 2: Kaplan-Meier survival plots presenting (a) OS and (b) DSS of the Lob/TT group and Lob/TT + CCND group for T1/T2 patients and (c) OS and (d) DSS of the TT group and TT + CCND group for T1/T2 patients treated with TT and RAI $(99 \times 87 \mathrm{~mm}(300 \times 300 \mathrm{DPI})$ ) 
fact that no previous research has provided direct evidence. Owing to the rarity of ITC, most published literatures are case reports or small institution-based case series. Studies with relatively sufficient number of cases rely more on population-level databases. The SEER program is a nationwide cancer database currently covering approximately 34.6 percent of the U.S. population [16]. More importantly, this database is available to cancer researchers worldwide, making it an important tool for clinical research across the globe, especially for rare tumors.

Although Conzo et al. reported that TT without routine prophylactic CCND, followed by RAI ablation also revealed low recurrence rate [11], we found that even with adjuvant RAI therapy, cN0 ITC patients without CCND still have significantly worse prognosis, suggesting that RAI ablation could not replace the therapeutic value of CCND in the treatment of this aggressive subtype. The generally low avidity for RAI in ITC might be a possible explanation. In a French cohort consisting of 104 PDTC patients (nearly 90\% were ITC), $50 \%(n=52)$ were identified to be RAI-refractory [17]. For patients receiving TT and RAI, absence of CCND demonstrated approximately 7 - and 15 -fold increased risk of all-cause and disease-specific mortality in the Type- $A$ and Type-B Cox models, respectively. We assumed that such high HRs was caused by the small number of deaths (3 allcause deaths, 1 due to ITC) among those receiving TT, RAI, and CCND.

Another important result derives from the subgroup analyses for T1/T2 disease. Although $P$ values of less than 0.05 could not be achieved because of the small samples, the non-negligible difference in the number of deaths between T1/T2 patients with and without CCND also prompted us to attach importance to the value of CCND in early-stage ITC patients.

As a most common subtype of PDTC, accurate preoperative diagnosis of ITC is crucial for planning optimal surgical management. However, its cytomorphological features are not well characterized, making it easily to be missed in fine-needle aspiration biopsy (FNAB) [18]. At present, it is mostly diagnosed by the pathology report of thyroidectomy specimens. Although Kane and Sharma tried to define the cytopathological features of PDTC in FNAB, more collaborative efforts are necessary to develop standardized cytologic diagnostic criteria [18]. It was also reported that the next-generation sequencing approach was useful to detect genetic biomarkers of PDTC and ATC [19]. However, this technique had a distance to go before its wide use in clinical practice.

A noteworthy point is that there are only 10 eligible pN1a patients in our study cohort despite the aggressive behavior of ITC. In a study using NCDB data, only 27 of 405 M0 ITC cases were diagnosed with N1a disease [2]. When we reviewed our selection process shown in Supplementary Figure S1, we found a higher proportion of patients were recorded as "M1/MX" $(n=43)$ or "N1b/N1-NOS/NX" $(n=33)$, suggesting that ITC tends to involve more extensive regions and disease confined to the central compartment is not as common as it is in PTC.
Some limitations should be acknowledged. First, as mentioned above, whether the $\mathrm{pN} 0 / \mathrm{pN} 1$ a patients were $\mathrm{cN} 0$ or $\mathrm{cN} 1$ was unknown in SEER database, so some patients in the thyroid-surgery + CCND group might undergo therapeutic CCND. Second, surgical margins were not recorded, although the thyroid-surgery + CCND group revealing better prognosis had a significantly higher proportion of patients with extrathyroidal extension, which sometimes lead to a difficulty in R0 resection. Third, dose of RAI, BRAF mutational status, and comorbidities were not provided in the database. Fourth, age at diagnosis of the CCND and NoCCND groups was not totally the same, which possibly influenced prognosis to some extent. However, in this study, the various multivariate Cox analyses for the overall cohort or subgroups might reduce the confounding effect of patients' age to a large extent. Fifth, this study is limited by its retrospective nature.

In conclusion, treating $\mathrm{CNO}$ ITC without CCND is associated with decreased survival compared with $\mathrm{pN} 0 / \mathrm{pN} 1 \mathrm{a}$ patients receiving CCND irrespective of $T$ staging and administration of adjuvant RAI therapy. Therefore, if a cN0 patient is diagnosed as ITC, after fully weighing the benefits and risks, prophylactic CCND may be considered as a secondary procedure (if postoperatively diagnosed) or a primary procedure (if intraoperatively/preoperatively diagnosed), especially for experienced high-volume surgeons. If possible, prospective studies are expected to validate our findings.

\section{Data Availability}

The data used to support the findings of this study are included within the article and within the supplementary information files.

\section{Conflicts of Interest}

The authors have no conflicts of interest to declare.

\section{Authors' Contributions}

Peng-Cheng Yu, Xiao Shi, Ben Ma, and Cui-Wei Li contributed equally to this work.

\section{Acknowledgments}

This study was supported by the National Science Foundation of China (81772851 to Yu-Long Wang and 81572622 to Qing-Hai Ji).

\section{Supplementary Materials}

Supplementary Figure S1: flow diagram representing the selection process. Supplementary Table S1: Type-B multivariate Cox regression models investigating the factors associated with OS and DSS in the overall cohort $(N=112)$. Supplementary Table S2: Type-B multivariate Cox regression models investigating the factors associated with OS and DSS for patients treated with TT $(N=98)$. Supplementary Table S3: Type-A multivariate Cox regression models investigating 
the factors associated with OS and DSS for patients treated with TT + RAI $(N=61)$. Supplementary Table S4: Type-B multivariate Cox regression models investigating the factors associated with OS and DSS for patients treated with TT + RAI $(N=61)$. Supplementary Table S5: Type-A multivariate Cox regression models investigating the factors associated with OS and DSS for patients treated without RAI therapy $(N=42)$. Supplementary Table S6: Type-B multivariate Cox regression models investigating the factors associated with OS and DSS for patients treated without RAI therapy $(N=42)$. Supplementary Table S7: Type-A multivariate Cox regression models investigating the factors associated with OS and DSS for patients with T3/T4-stage $(N=77)$. Supplementary Table S8: Type-B multivariate Cox regression models investigating the factors associated with OS and DSS for patients with T3/ T4-stage $(N=77)$. Supplementary Table S9: baseline characteristics of patients with T1/T2 tumors $(N=35)$. (Supplementary Materials)

\section{References}

[1] M. L. Carcangiu, G. Zamp, and J. Rosai, "Poorly differentiated ("insular") thyroid carcinoma," The American Journal of Surgical Pathology, vol. 8, no. 9, pp. 655-668, 1984.

[2] T. A. Pezzi, V. C. Sandulache, C. M. Pezzi et al., "Treatment and survival of patients with insular thyroid carcinoma: 508 cases from the National Cancer Data Base," Head \& Neck, vol. 38, no. 6, pp. 906-912, 2016.

[3] B. R. Haugen, E. K. Alexander, K. C. Bible et al., "2015 American thyroid association management guidelines for adult patients with thyroid nodules and differentiated thyroid cancer: the American thyroid association guidelines task force on thyroid nodules and differentiated thyroid cancer," Thyroid, vol. 26, no. 1, pp. 1-133, 2016.

[4] M. Volante and M. Papotti, "Poorly differentiated thyroid carcinoma: 5 years after the 2004 WHO classification of endocrine tumours," Endocrine Pathology, vol. 21, no. 1, pp. 1-6, 2010.

[5] H. S. Kazaure, S. A. Roman, and J. A. Sosa, "Insular thyroid cancer," Cancer, vol. 118, no. 13, pp. 3260-3267, 2012.

[6] L. Falvo, A. Catania, V. D’Andrea, P. Grilli, C. D’Ercole, and E. De Antoni, "Prognostic factors of insular versus papillary/ follicular thyroid carcinoma," American Surgeon, vol. 70, no. 5, pp. 461-466, 2004.

[7] A. Agha, G. Glockzin, M. Woenckhaus, W. Dietmaier, I. Iesalnieks, and H. J. Schlitt, "Insular carcinomas of the thyroid exhibit poor prognosis and long-term survival in comparison to follicular and papillary T4 carcinomas," Langenbeck's Archives of Surgery, vol. 392, no. 6, pp. 671-677, 2007.

[8] M. Papotti, F. B. Micca, A. Favero, N. Palestini, and G. Bussolati, "Poorly differentiated thyroid carcinomas with primordial cell component," The American Journal of Surgical Pathology, vol. 17, no. 3, pp. 291-301, 1993.

[9] National Comprehensive Cancer Network, Clinical Practice Guidelines in Oncology: Thyroid Carcinoma, National Comprehensive Cancer Network, Fort Washington, PA, USA, 2017, http://www.nccn.org/professionals/physician_gls/PDF/ thyroid.pdf.2017.

[10] National Comprehensive Cancer Network, Clinical Practice Guidelines in Oncology: Thyroid Carcinoma, National
Comprehensive Cancer Network, Fort Washington, PA, USA, 2018, http://www.nccn.org/professionals/physician_gls/PDF/ thyroid.pdf.2018.

[11] G. Conzo, D. Pasquali, G. Bellastella et al., "Total thyroidectomy, without prophylactic central lymph node dissection, in the treatment of differentiated thyroid cancer. Clinical retrospective study on 221 cases," Endocrine, vol. 44, no. 2, pp. 419-425, 2013.

[12] D. T. Hughes, M. L. White, B. S. Miller, P. G. Gauger, R. E. Burney, and G. M. Doherty, "Influence of prophylactic central lymph node dissection on postoperative thyroglobulin levels and radioiodine treatment in papillary thyroid cancer," Surgery, vol. 148, no. 6, pp. 1100-1106, 2010.

[13] X. Shi, N.-S. Huang, B.-W. Lei et al., "Central lymph node status has significant prognostic value in the clinically nodenegative tall-cell variant of papillary thyroid cancer regardless of $T$-staging and radioactive iodine administration: first evidence from a population-based study," Annals of Surgical Oncology, vol. 25, no. 8, pp. 2316-2322, 2018.

[14] E.-J. Wagenmakers and S. Farrell, "AIC model selection using Akaike weights," Psychonomic Bulletin \& Review, vol. 11, no. 1, pp. 192-196, 2004.

[15] D. Posada and T. R. Buckley, "Model selection and model averaging in phylogenetics: advantages of akaike information criterion and bayesian approaches over likelihood ratio tests," Systematic Biology, vol. 53, no. 5, pp. 793-808, 2004.

[16] National Cancer Institute, Surveillance, Epidemiology, and End Results (SEER) Program. Overview of the SEER Program, National Cancer Institute, Bethesda, MD, USA, 2016, https://seer.cancer.gov/about/overview.html.

[17] C. de la Fouchardière, M. Decaussin-Petrucci, J. Berthiller et al., "Predictive factors of outcome in poorly differentiated thyroid carcinomas," European Journal of Cancer, vol. 92, pp. 40-47, 2018.

[18] S. V. Kane and T. P. Sharma, "Cytologic diagnostic approach to poorly differentiated thyroid carcinoma: a single-institution study," Cancer Cytopathology, vol. 123, no. 2, pp. 82-91, 2015.

[19] V. Sykorova, S. Dvorakova, J. Vcelak et al., "Search for new genetic biomarkers in poorly differentiated and anaplastic thyroid carcinomas using next generation sequencing," Anticancer Research, vol. 35, no. 4, pp. 2029-2036, 2015. 


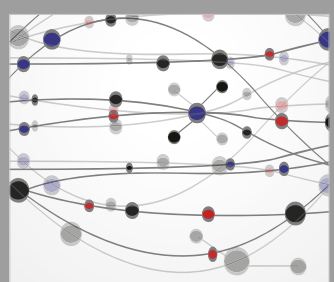

The Scientific World Journal
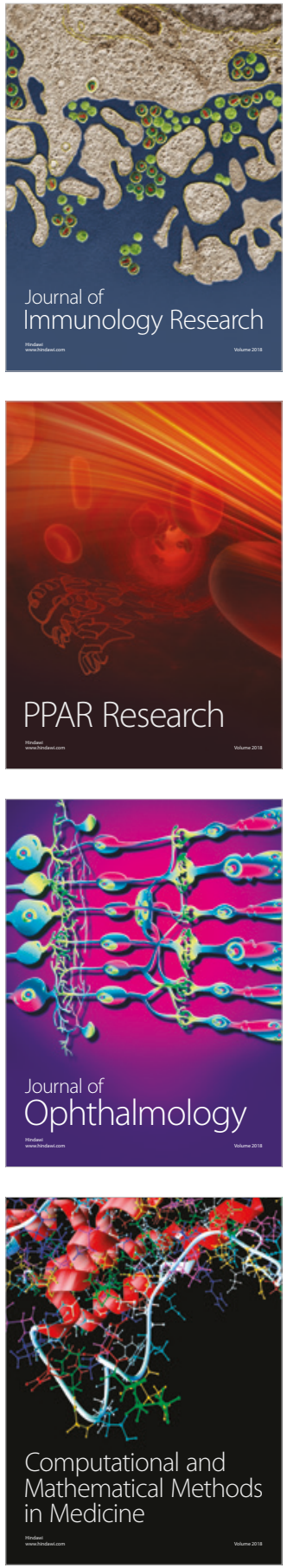

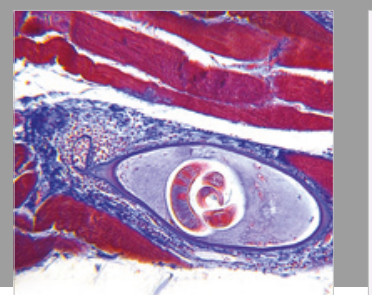

Gastroenterology Research and Practice

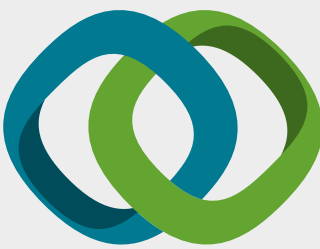

\section{Hindawi}

Submit your manuscripts at

www.hindawi.com
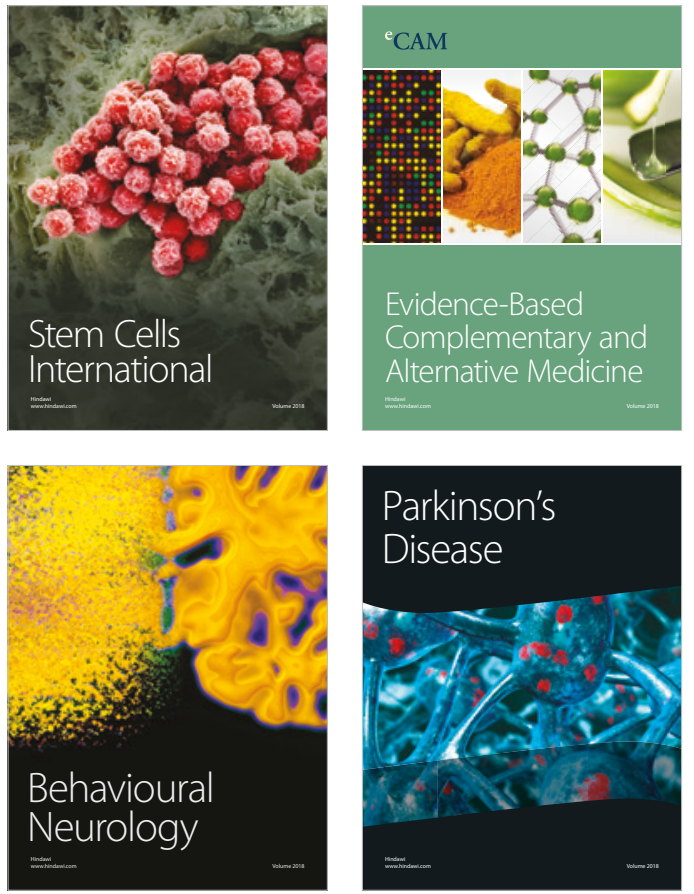

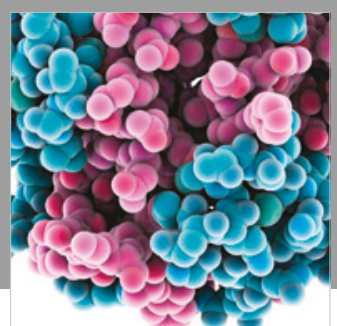

ournal of

Diabetes Research

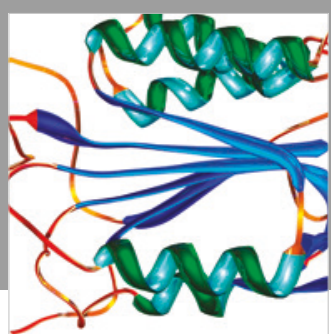

Disease Markers
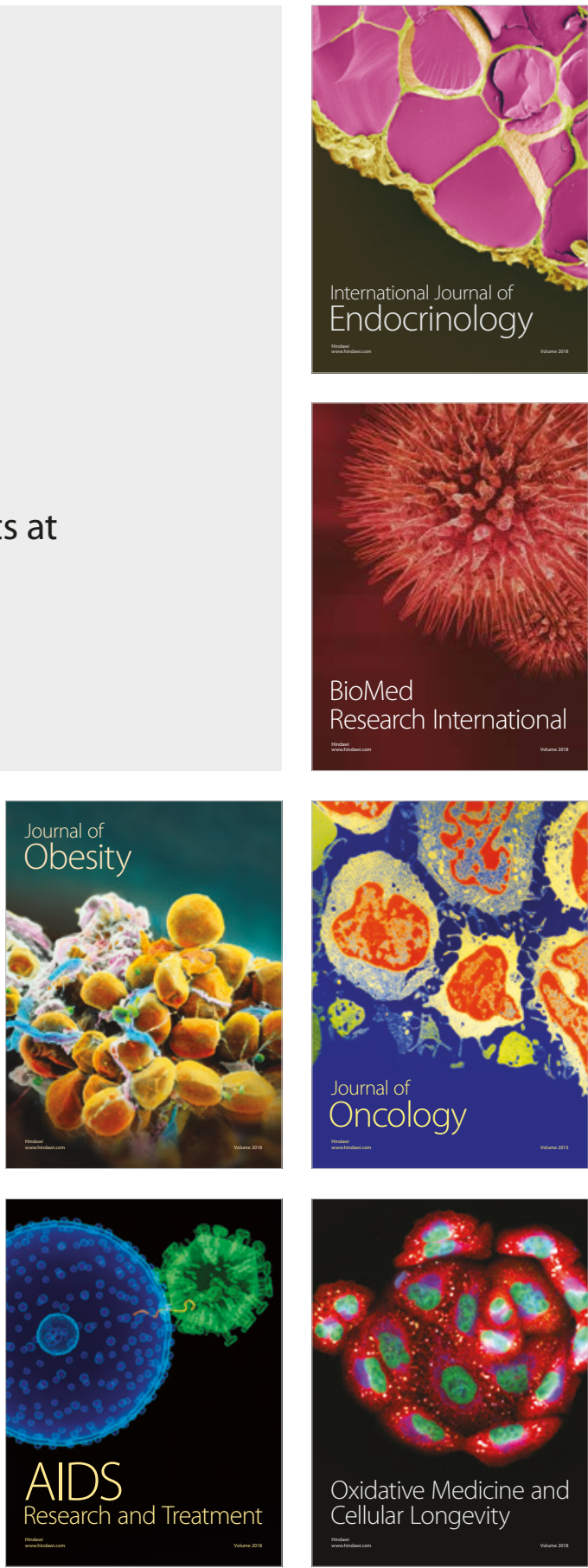